\title{
侧链含不饱和基团取代的硫代双烯型镍络合物的合成及其性能
}

\author{
孙健 ${ }^{a}$ 金哲明 $b$ 王成云 ${ }^{a}$ 沈永嘉*,a \\ ( ${ }^{a}$ 华东理工大学精细化工研究所 结构可控先进功能材料及其制备教育部重点实验室 上海 200237) \\ $\left({ }^{b}\right.$ 朝鲜民主主义人民共和国咸兴化工大学 朝鲜)
}

\begin{abstract}
摘要 合成了 3 个侧链含不饱和基团取代的硫代双烯型镍络合物, 即双 [4,5-二(4'-叔丁基苄基)硫-1,3-二硫杂环戊烯]镍 (2a)、双 [4,5-二(烯丙基)硫-1,3-二硫杂环戊烯]镍(2b)和双 [4,5-二(3'-苯基-烯丙基)硫-1,3-二硫杂环伐烯]镍(2c) 以及作为参 照品的一个侧链含饱和基团的硫代双烯型镍络合物, 即双 [4,5-二(癸基)硫-1,3-二硫杂环戊烯]镍(2d). 它们的结构经 ${ }^{1} \mathrm{H}$ NMR 和质谱得到了表征. 这些化合物在甲苯中有较好的溶解性，最大吸收波长 $\left(\lambda_{\max }\right)$ 在 $1000 \mathrm{~nm}$ 左右，而且取代基对它 们最大吸收波长的位移影响很小. 它们在固态聚甲基丙烯酸甲酯(PMMA)中的 $\lambda_{\max }$ 也在 $1000 \mathrm{~nm}$ 左右, 且浓度较低时, 吸收曲线未见有宽化的迹象. 固态循环伏安的测试结果表明, 苯基的引入对提高这类化合物的氧化电位有影响. 热重 分析(TGA)的结果表明, 这类化合物失重 $5 \%$ 的温度都在 $200{ }^{\circ} \mathrm{C}$ 以上. 氙灯 $(300 \mathrm{~W})$ 照射 $100 \mathrm{~min}$, 它们的吸光度在甲苯 中下降不到 $10 \%$, 在固态的 PMMA 中下降不到 $2 \%$ ，苯基的引入有助于提高这类化合物在溶液和固态 PMMA 中的光化 学稳定性.
\end{abstract}

关键词＼cjkstart硫代双烯型镍络合物; 合成; 近红外吸收; 光稳定性; 热稳定性

\section{Synthesis and Properties of Extended Bis-1,2-dithiolene Nickel Complexes Substituted with Unsaturated Group}

\author{
Sun, Jian ${ }^{a} \quad$ Jin, Zheming ${ }^{b} \quad$ Wang, Chengyun $^{a} \quad$ Shen, Yongjia ${ }^{*, a}$ \\ ( ${ }^{a}$ Key Laboratory for Advanced Materials and Institute of Fine Chemicals, East China University of \\ Science \& Technology, Shanghai 200237) \\ ( ${ }^{b}$ Xianxing Chemical Industry University, Democratic People's Republic of Korea)
}

\begin{abstract}
Three bis-1,2-dithiolene nickel complexes with unsaturated substituted groups in the terminals, bis[4,5-di(4'-tbutylbenzyl)thio-1,3-dithion]nickel (2a), bis[4,5-dially-thio-1,3-dithion]nickel (2b) and bis[4,5-di(3'-phenyl-ally)thio-1,3-dithion]nickel (2c) together with a bis-1,2-dithiolene nickel complexes with saturated substituted groups in the terminals, bis[4,5-didecylthio-1,3-dithion]nickel (2d), as a reference, were synthesized and characterized by ${ }^{1} \mathrm{H}$ NMR and MS. They were easily soluble in toluene with the maximum absorption wavelengths $\left(\lambda_{\max }\right)$ around $1000 \mathrm{~nm}$, the effects of substituent groups in the terminals on $\lambda_{\max }$ were not so significant. Their $\lambda_{\max }$ in solid polymethyl methacrylate (PMMA) were around $1000 \mathrm{~nm}$ as well, and no broadening absorption bands could be observed in lower concentration. The solid cyclic voltammetry (CV) curves showed that the oxidation potentials would be increased by introduction of phenyl group in the terminals, thus their electrochemical stability could be improved. The results of thermal gravimetric analysis (TGA) indicated that the temperature of $5 \%$ weight-loss was higher than $200{ }^{\circ} \mathrm{C}$ for these compounds. Irradiated by xenon lamp $(300 \mathrm{~W})$ for 100 min, their absorbance was decreased less than $100 \%$ in toluene and $2 \%$ in solid polymethyl methacrylate (PMMA), respectively. The introduction of phenyl group in the terminals could improve the photo-stability for these molecules both in solution and solid PMMA.
\end{abstract}

Keywords bis-1,2-dithiolene nickel complexes; synthesis; near-infrared absorption; photo-stability; thermal stability

硫代双烯型金属络合物作为近红外吸收染料中的 一个较新的成员 ${ }^{[1-3]}$, 自 20 世纪一问世就受到关注, 因 为它们的吸收波长范围在 $600 \sim 1600 \mathrm{~nm}$, 是已知的近 红外染料中吸收波长最长的, 同时它们在非极性有机溶
剂中也比同类有更高的溶解度 ${ }^{[4,5]}$, 这些非常有利于它 们在军事方面和远程通讯方面的应用. 理论上，该类化 合物中的配位金属离子可以是任何过渡金属离子, 但是 研究较多的是镍、钯、铂的络合物 ${ }^{[6]}$. 根据这类化合物

\footnotetext{
*E-mail: yjshen@ecust.edu.cn

Received March 4, 2015; revised May 5, 2015; published online May 14, 2015.
} 
的结构可以将它们细分为两类, 见图 1, 通过修饰分子 侧链上的取代基, 可以调节它们的最大吸收波长. 如今, 硫代双烯型金属络合物(尤其是硫代双烯型镍络合物), 作为近红外吸收材料, 在非线性光学材料 ${ }^{[7]}$ 、导电材 料 ${ }^{[8,9]}$ 和磁性材料 ${ }^{[10,11]}$ 得到了应用或是应用研究. 国内 的学者, 如, 程铸生 ${ }^{[12]} 、 门$ 金风 ${ }^{[13]}$ 、戴峰 ${ }^{[14]}$ 和高俊雄 ${ }^{[15]}$ 等, 曾经合成过一些结构如 Scheme 1 所示的硫代双烯 型镍络合物, 并研究了将它们作为红外激光调 $\mathrm{Q}$ 染 料 ${ }^{[12]}$ 、单线态氧猝灭剂 ${ }^{[13]}$ 和夜视兼容近红外滤光 片 $^{[14]}$ 的性能. 国外的学者研究过 2 所示硫代双烯型镍络合物 的合成与性能, 它们中, 侧链 $\mathrm{SR}$ 中的 $\mathrm{R}$ 基团主要是饱 和脂肪链 ${ }^{[16]}$. 本文设计了 4 个结构的硫代双烯型镍络合 物 2(图 2), 其中, 有三个的侧链上含不同形式的不饱和 取代基. 这里报道它们的合成以及它们的光物理和光化 学性能以及电化学和热化学性能.
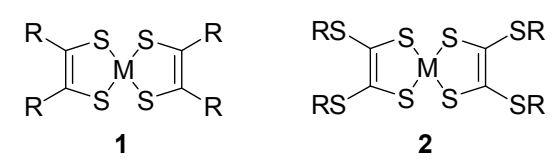

图 1 硫代双烯型络合物分子结构

Figure 1 The generic molecular structure of the transition metal dithiolene core

$$
\underset{\mathrm{MeOH}_{2} \mathrm{Na}, \mathrm{NBu}_{4} \mathrm{Br}}{\mathrm{NiCl}_{2} \cdot 6 \mathrm{H}_{2} \mathrm{O}, \mathrm{I}_{2}, \mathrm{CH}_{3} \mathrm{CN}}
$$

图式 1 化合物 2 的合成路线

Scheme 1 the synthetic process of compound $\mathbf{2}$

\section{2 结果与讨论}

\section{1 化合物 $2 \mathrm{a} \sim 2 \mathrm{~d}$ 的合成}

合成化合物 $\mathbf{2 a} \sim \mathbf{2 d}$ 的反应式如 Scheme 1 所示.

化合物 $\mathbf{2 a} \sim 2 \mathbf{d}$ 的合成分为两步 ${ }^{[17]}$ : 第一步反应以 锌酸盐 (bis(tetraethylammonium)-bis(1,3dithiol-(2)-thion4,5-dithiolato) Zinc, Zincate) 3 为起始原料, 它是合成四 硫代富瓦烯类化合物的基础原料. 锌酸盐在丙酮或乙腈 中极易与活泼的卤代烃发生反应, 生成一种称为硫酮 (dithione)的化合物 4. 由于在合成 3 的过程中会不可避 免地生成一个副产物, 它很难与 3 分离 ${ }^{[18]}$, 但是在与卤 代烃反应后, 混杂在 $\mathbf{4}$ 中的副产物很容易用硅胶柱层析 的方法分离. 含癸基的硫酮 $4 \mathrm{~d}$ 由于溶解度好, 用石油醚 为洗脱剂即可与其副产物分离. 含 4-叔丁基茮基、丙烯 基和 3-苯基丙烯基的硫酮 $\mathbf{4 a} \sim \mathbf{4 c}$ 的硅胶柱层析需要用

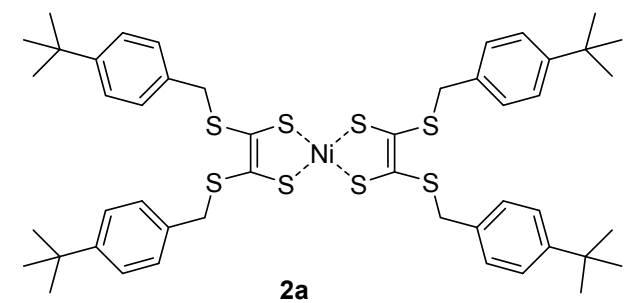

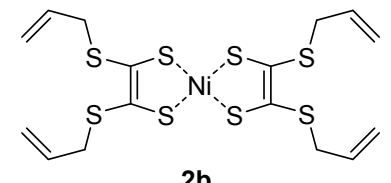

2b

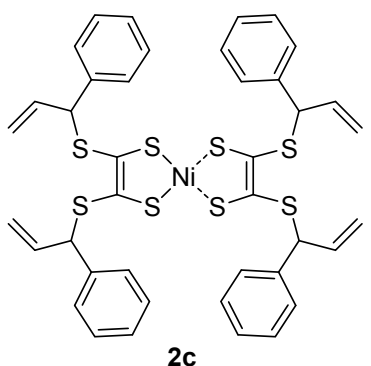

$\mathrm{C}_{10} \mathrm{H}_{21} \mathrm{~S} \longrightarrow \mathrm{S}^{\prime} \mathrm{Ci}^{\prime} \mathrm{H}_{21} \mathrm{~S} \mathrm{SC}_{10} \mathrm{H}_{21}$

2d

图 2 化合物 $\mathbf{2 a} \sim 2 \mathrm{~d}$ 的分子结构

Figure 2 Structures of compound $\mathbf{2 a} \sim \mathbf{2 d}$

石油醚/二氯甲烷作为洗脱剂. 合成化合物 $\mathbf{2 a} \sim \mathbf{2 d}$ 的关 键在于第二步, 从硫酮 $\mathbf{4 a} \sim \mathbf{4 d}$ 到化合物 $\mathbf{2 a} \sim \mathbf{2 d}$ 看似是 一步反应，事实上它经历了 3 个步骤，如 Scheme 2 所示.

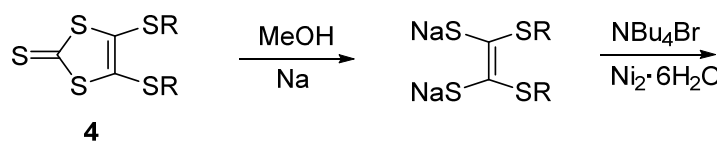

图式 2 生成化合物 $\mathbf{2}$ 的机理

Scheme 2 The mechanism of forming compound 2

硫酮在甲醇钠的作用下首先形成了一个钠盐，这个 阶段的反应比较容易控制, 通过薄板层析(TLC)即可判 断反应的终点. 钠盐在同一反应介质中与 $\mathrm{NiCl}_{2}$ 反应生 成季铵盐的反应不容易控制, 因为产物是一个活泼的阴 离子, 故一般是在加入 $\mathrm{NiCl}_{2}$ 的同时也加入 $\mathrm{Bu}_{4} \mathrm{NBr}$, 它 的作用是提供一个阳离子以平衡产物的电中性, 使之稳 定. 该阶段的反应产物可以从反应体系中分离出 来 ${ }^{[16,19]}$. 为简化反应的操作, 本文在该阶段通过减压蒸 馏除去甲醇, 然后将残余物溶解在乙腈中进行后续的氧 化反应. 这一举措的好处在于化合物 $\mathbf{2 a} \sim \mathbf{2 d}$ 在乙腈中 的溶解度较小, 这样氧化产物在乙腈中可以很容易地以 固体的形式从反应介质中析出, 使得反应的后处理容 易，同时也可提高化合物 $\mathbf{2 a} \sim \mathbf{2 d}$ 的产率. 


\section{2 化合物 $2 \mathrm{a} \sim 2 \mathrm{~d}$ 的光吸收行为}

\subsection{1 在溶液中的光吸收行为}

化合物 $\mathbf{2} \mathbf{a} \sim 2 \mathrm{~d}$ 在丙酮、二氯甲烷、甲苯和四氯乙 烯中有较好的溶解性, 作为代表, 化合物 $2 \mathrm{a}$ 在这四种溶 剂中的吸收光谱曲线见图 3.4 个化合物的最大吸收波长 $\left(\lambda_{\max }\right)$ 和摩尔消光系数见表 1 . 可见, 化合物 $\mathbf{2 a} \sim \mathbf{2 d}$ 的 $\lambda_{\max }$ 均在 $1000 \mathrm{~nm}$ 左右, 比起分子中含 4 个 $\mathrm{S}$ 的化合物 $\mathbf{1}, \lambda_{\max }$ 红移了 $500 \mathrm{~nm}$ 以上 ${ }^{[13,15]}$.

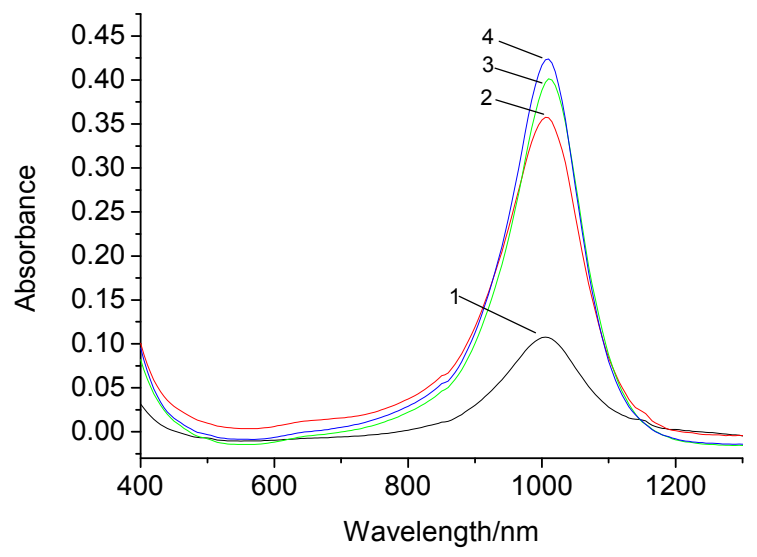

图 3 化合物 $2 \mathrm{a}$ 在丙酮(1)、二氯甲烷(2)、甲苯(3)和四氯乙烯 (4)中的吸收光谱

Figure 3 Absorption spectra of compound 2a in acetone (1), dichloromethane (2), toluene (3) and tetrachlorethylene (4)

表 1 的数据表明, 取代基的不同对化合物 $\mathbf{2 a} \sim \mathbf{2 d}$ 的 $\lambda_{\text {max }}$ 无甚影响, 甚至溶剂的极性不同对化合物 $\mathbf{2 a} \sim 2 \mathbf{d}$ 的 $\lambda_{\text {max }}$ 也无甚影响, 这与文献报道的化合物 $\mathbf{1}$ 的光吸收 行为不同 ${ }^{[13]}$. 通常, 取代基的供电性不同会造成衍生物 的 $\lambda_{\text {max }}$ 发生红移或蓝移. 这里, 取代基不同对化合物 $\mathbf{2 a} \sim 2 \mathbf{d}$ 的 $\lambda_{\text {max }}$ 影响之所以不大, 是因为侧链上 $\mathrm{S}$ 原子的 缘故. $\mathrm{S}$ 原子上有两对未共享电子对, 所以它的供电性 很强, 以致其后面的烷基或苯基的供电性强弱对母体的 影响小到几乎没有.

此外, 表 1 的数据还表明, 化合物 $\mathbf{2 a} \sim \mathbf{2 d}$ 在丙酮中 的吸收强度均较低, 对化合物 $\mathbf{2 b}$ 和 $2 \mathrm{c}$ 而言, 它们在丙 酮中的吸收强度比它们在另三种溶剂中的吸收强度要 小一个数量级. 本以为是测量误差, 但经反复测试后发 现并非如此，它们在丙酮中的吸收确实比较弱.

2.2.2 在聚甲基丙烯酸甲酯(PMMA)中的光吸收行为 近红外吸收染料的一个重要用途是制作近红外光 滤色片, 所以研究硫代双烯型金属镍络合物在聚合物中 的光吸收行为非常重要. 本文在同等质量的聚甲基丙烯 酸甲酯(PMMA)中，加入不同量的化合物 $\mathbf{2 a} \sim \mathbf{2 d}$ 制得了 一系列厚度相同、表面积一样的近红外滤光片. 作为代 表, 不同含量的化合物 $\mathbf{2 a}$ 在 PMMA 中的吸收曲线见图
表 1 化合物 $2 \mathrm{a} \sim 2 \mathrm{~d}$ 在丙酮、二氯甲烷、甲苯和四氯乙烯中 的最大吸收波长和摩尔消光系数

Table 1 The maximum absorption wavelengths and molar extinguish coefficients of compounds $\mathbf{2 a} \sim \mathbf{2 d}$ in acetone, dichloromethane, toluene and tetrachlorethylene

\begin{tabular}{|c|c|c|c|}
\hline 化合物 & 溶剂 & 最大吸收波长 $/ \mathrm{nm}$ & $\begin{array}{l}\text { 摩尔消光系数/ } \\
\left(\mathrm{L} \cdot \mathrm{mol}^{-1} \cdot \mathrm{cm}^{-1}\right)\end{array}$ \\
\hline \multirow{4}{*}{$2 a$} & 丙酮 & 1000 & $1.078 \times 10^{4}$ \\
\hline & 四氯乙烯 & 1005 & $4.237 \times 10^{4}$ \\
\hline & 二氯甲烷 & 1010 & $3.577 \times 10^{4}$ \\
\hline & 甲苯 & 1010 & $4.013 \times 10^{4}$ \\
\hline \multirow{4}{*}{$2 b$} & 丙酮 & 1000 & $4.590 \times 10^{3}$ \\
\hline & 四氯乙烯 & 1000 & $1.502 \times 10^{4}$ \\
\hline & 二氯甲烷 & 1000 & $1.501 \times 10^{4}$ \\
\hline & 甲苯 & 1000 & $1.860 \times 10^{4}$ \\
\hline \multirow{4}{*}{$2 \mathrm{c}$} & 丙酮 & 1005 & $8.030 \times 10^{3}$ \\
\hline & 四氯乙烯 & 1005 & $2.955 \times 10^{4}$ \\
\hline & 二氯甲烷 & 1010 & $9.868 \times 10^{4}$ \\
\hline & 甲苯 & 1010 & $2.777 \times 10^{4}$ \\
\hline \multirow{4}{*}{ 2d } & 丙酮 & 1000 & $2.599 \times 10^{4}$ \\
\hline & 四氯乙烯 & 1000 & $2.623 \times 10^{4}$ \\
\hline & 二氯甲烷 & 1005 & $5.403 \times 100^{4}$ \\
\hline & 甲苯 & 1005 & $2.605 \times 10^{4}$ \\
\hline
\end{tabular}

4, 化合 $\mathbf{2 a} \sim \mathbf{2 d}$ 在 PMMA 中的吸收数据见表 2 .

表 2 的数据表明, 不同含量的化合物 $\mathbf{2 a} \sim \mathbf{2 d}$ 在 PMMA 中的最大吸收峰值出现在 $1000 \sim 1010 \mathrm{~nm}$ 之间, 这与它们在溶液中的吸收波长相近. 浓度较低时, 吸收 曲线较为光滑, 说明低浓度的化合物 $\mathbf{2 a} \sim \mathbf{2 d}$ 与 PMMA 结合后, 它们在 PMMA 中是以分子状态分散在其中, 且 溶质分子与溶剂中没有发生团聚，是一种固态溶液. 浓 度较高时, 吸收曲线不光滑, 说明化合物 $\mathbf{2 a} \sim \mathbf{2 d}$ 在 PMMA 中发生了分子间的团聚. 照理, 在溶剂或介质 中, 分子发生团聚后, 其吸收峰会变宽, 最大吸收峰值 也会红移，但是图 4 中的吸收曲线未见有宽化的迹象， 也未见最大吸收峰值发生变化. 这说明虽然化合物 $\mathbf{2 a} \sim 2 \mathbf{d}$ 分子在 PMMA 发生团聚, 但是毕竟其浓度不高, 因而团聚程度也不高, 故对吸收曲线的形状和波长范围 影响甚小. 此外, 化合物 $\mathbf{2 a} \sim \mathbf{2 d}$ 与 PMMA 结合后形成 的近红外滤光片, 在可见光区域几乎没有吸收, 即使化 合物 $\mathbf{2 a} \sim 2 \mathbf{d}$ 的浓度增加到 $0.04 \%$, 在可见光区域仍无明 显的吸收. 这说明, 由化合物 $\mathbf{2 a} \sim \mathbf{2 d}$ 与 PMMA 制成的 滤光片在阻隔近红外光线的同时，对可见光区域波段吸 收较小, 这一点对于将它们制成护目镜之类的防护红外 激光的器械非常有利.

\section{3 化合物 $2 \mathrm{a} \sim 2 \mathrm{~d}$ 的电化学性能}

为了研究不同取代基对化合物 $\mathbf{2 a} \sim \mathbf{2 d}$ 电化学性能 的影响, 通过循环伏安法测得了化合物 $\mathbf{2 a} \sim \mathbf{2 d}$ 在固态 


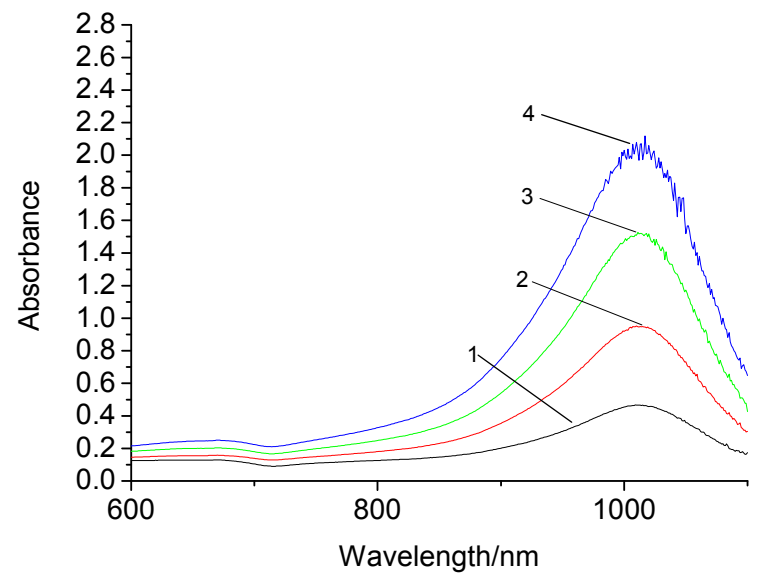

图 4 不同含量的化合物 2a 在 PMMA 中的吸收光谱

Figure 4 Absorption spectra of 2a in PMMA depending on its concentration

(1) $0.01 \mathrm{wt} \%$, (2) $0.02 \mathrm{wt} \%$, (3) $0.03 \mathrm{wt} \%$, (4) $0.04 \%$

表 2 化合物 $\mathbf{2 a} \sim \mathbf{2 d}$ 在 PMMA 中的光吸收数据

Table 2 Absorption data of compounds $\mathbf{2 a} \sim \mathbf{2 d}$ in PMMA

\begin{tabular}{ccc}
\hline 化合物 & 质量分数 $/ \mathrm{wt} \%$ & 最大吸收波长 $/ \mathrm{nm}$ \\
\hline \multirow{2}{*}{$\mathbf{2 a}$} & 0.01 & 1005 \\
& 0.02 & 1005 \\
& 0.03 & 1005 \\
& 0.04 & 1005 \\
& 0.01 & 1000 \\
$\mathbf{2 b}$ & 0.02 & 1000 \\
& 0.03 & 1000 \\
& 0.04 & 1000 \\
& 0.01 & 1010 \\
2c & 0.02 & 1010 \\
& 0.03 & 1010 \\
& 0.04 & 1010 \\
& 0.01 & 1005 \\
2d & 0.02 & 1005 \\
& 0.03 & 1005 \\
& 0.04 & 1005 \\
\hline
\end{tabular}

时的氧化电位, 它们的循环伏安图见图 5. 由图中的数 值利用计算公式(表 3)计算了它们的最高占有轨道 (HOMO) 和最低空轨道(LUMO) 能级值以及能带隙, 结 果见表 3.

表 3 的数据表明, 化合物 $\mathbf{2 a}$ 与 $2 \mathbf{d}$ 相比, $2 \mathbf{a}$ 的氧化 电位要高, 也就是 $\mathbf{2 a}$ 比 $\mathbf{2 d}$ 不容易氧化, 而 $\mathbf{2 a}$ 可以看作 是在饱和的脂肪链中引入了一个苯环. 化合物 $\mathbf{2 c}$ 与 $\mathbf{2 b}$ 相比, $2 \mathrm{~b}$ 的氧化电位要高一些. $2 \mathrm{c}$ 可看作是在不饱和脂 肪链中引入一个苯环. 上述事实说明, 苯基的引入对提 高硫代双烯型镍络合物的电化学稳定性有影响. 另一方 面, $2 \mathrm{a}$ 与 $2 \mathrm{~d}$ 相比, 能带隙的数值下降了 $0.24 \mathrm{eV} . \mathbf{2 c}$ 与 $\mathbf{2 b}$ 相比, 能带隙的数值也下降了 $0.15 \mathrm{eV}$. 而 $\mathbf{2 b}$ 与 $\mathbf{2 d}$

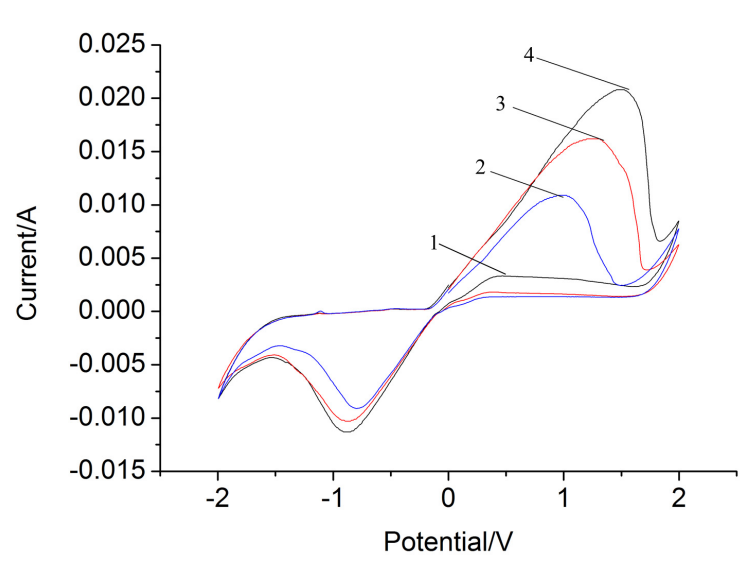

图 5 化合物 $\mathbf{2 a} \sim 2 \mathrm{~d}$ 的循环伏安曲线

Figure $5 \mathrm{CV}$ curves of compound $\mathbf{2 a} \sim \mathbf{2 d}$

(1) 2c, (2) 2b, (3) 2d, (4) 2a

表 3 化合物 $2 \mathrm{a} \sim 2 \mathrm{~d}$ 固态时的氧化电位与计算的最高占有轨 道和最低空轨道及能带隙数值

Table 3 Oxidation potentials and HOMO-LUMO and energy gap calculated of compounds $\mathbf{2 a} \sim \mathbf{2 d}$

\begin{tabular}{cclcc}
\hline Compd. & Eonset-ox ${ }^{a} / \mathrm{V}$ & $\mathrm{HOMO}^{b} / \mathrm{eV}$ & $E_{\mathrm{g}}^{c} / \mathrm{eV}$ & $\mathrm{LUMO}^{d} / \mathrm{eV}$ \\
\hline 2a & 1.49 & -6.19 & 2.61 & -3.58 \\
2b & 1.26 & -5.96 & 2.82 & -3.14 \\
2c & 00.98 & -5.68 & 2.67 & -2.66 \\
2d & 1.38 & -6.008 & 2.85 & -3.23 \\
\hline
\end{tabular}

${ }^{a}$ Eonset-ox $=$ onset oxidation potential measured by cyclic voltammetry. ${ }^{b} \mathrm{HOMO}=-\mathrm{e}($ Eonset-ox +4.7$) .{ }^{c} E_{\mathrm{g}}$ estimated from the UV-Vis absorption spectra. ${ }^{d} \mathrm{LUMO}=E_{\mathrm{g}}+\mathrm{HOMO}$.

的能带隙值仅相差 $0.03 \mathrm{eV}$. 这表明, 不饱和基团的引入 可以降低硫代双烯型镍络合物的能带隙，但是它的影响 程度不如引入苯环那样明显.

\section{4 化合物 $2 \mathrm{a} \sim 2 \mathrm{~d}$ 的热稳定性能}

在将化合物 $\mathbf{2 a} \sim \mathbf{2 d}$ 用于制作近红外吸收滤光片的 过程中, 依加工方式和聚合物种类的不同, 需要在高温 下进行, 例如, 当滤色片的基材是聚碳酸酯( $\mathrm{PC}$ )时, 其 加工温度在 $260 \sim 300{ }^{\circ} \mathrm{C}^{[20]}$. 为此, 要求作为近红外吸 收材料使用的化合物具有相应的耐热稳定性. 因为若化 合物的分解温度低于滤色片的加工温度, 则它会在加工 过程中分解，从而失去了近红外吸收能力. 化合物 $\mathbf{2 a} \sim$ 2d 的 TGA 测试在氮气保护下进行, 升温速率为 $10{ }^{\circ} \mathrm{C} /$ $\min$.

图 6 是化合物 $\mathbf{2 a} \sim \mathbf{2 d}$ 的 TGA 曲线, 表 4 是它们失 重 $5 \%$ 的温度和在 $300{ }^{\circ} \mathrm{C}$ 时的质量损失. 由表 4 的数据 可见, 化合物 2a $2 \mathrm{~d}$ 均有良好的热稳定性, 失重 5\%的 温度均大于 $200{ }^{\circ} \mathrm{C}$, 其中, 含 4-叔丁基苄基的化合物 $\mathbf{~ c c}$ 不仅失重 $5 \%$ 的温度最高, 达到 $273{ }^{\circ} \mathrm{C}$, 而且当温度达 到 $300{ }^{\circ} \mathrm{C}$ 时, 仅失重 $11.5 \%$. 另一个含苯基的化合物 $2 \mathrm{a}$ 其分解温度也较高, 失重 $5 \%$ 的温度在 $234{ }^{\circ} \mathrm{C}$, 它们均 
比不含苯基的化合物 $\mathbf{2 b}$ 和 $\mathbf{2 d}$ 要高出 $20{ }^{\circ} \mathrm{C}$ 以上. 两个 不含苯基的化合物失重 $5 \%$ 的温度只相差 $3{ }^{\circ} \mathrm{C}$, 由此可 见, 苯基的引入对提高此类化合物的热稳定性能起着关 键的作用.

表 4 化合物 $\mathbf{2 a} \sim 2 \mathrm{~d}$ 的 TGA 数据

Table 4 TGA of compounds $\mathbf{2 a} \sim \mathbf{2 d}$

\begin{tabular}{ccc}
\hline 化合物 & 失重 $5 \%$ 的温度 $/{ }^{\circ} \mathrm{C}$ & 质量损失 $\left(300{ }^{\circ} \mathrm{C}\right) / \%$ \\
\hline $\mathbf{2 a}$ & 234 & 40.00 \\
$\mathbf{2 b}$ & 210 & 32.81 \\
$\mathbf{2 c}$ & 273 & 11.49 \\
$\mathbf{2 d}$ & 213 & 68.58 \\
\hline
\end{tabular}

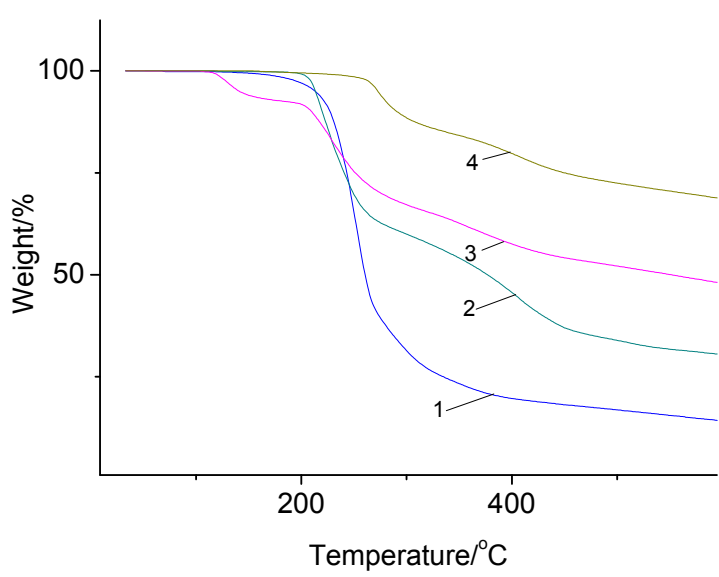

图 6 化合物 $\mathbf{2 a} \sim \mathbf{2 d}$ 的 TGA 曲线

Figure 6 TGA curves of compound $\mathbf{2 a} \sim \mathbf{2 d}$ (1) 2d, (2) 2a, (3) 2b, (4) 2c

\section{4 化合物 $2 \mathrm{a} \sim 2 \mathrm{~d}$ 的光化学稳定性}

\subsection{1 在溶液中的光化学稳定性}

以氙灯为光源, 测试了化合物 $\mathbf{2 a} \sim 2 \mathbf{d}$ 在甲苯中(浓 度： $1.00 \times 10^{-4} \mathrm{~mol} / \mathrm{L}$ )的吸光度随光照时间的变化, 以 此考察它们的光化学稳定性, 结果见图 7. 总体而言, 这 4 个化合物的光稳定性都较好, 光照 $100 \mathrm{~min}$, 它们中 光化学稳定性最差的(侧链中含烯丙基的 $2 \mathbf{b}$ ) 吸光度下 降不到 10\%; 光化学稳定性最好的(侧链中含苯基烯丙 基的 2c) 吸光度下降不到 2\%. 另一个分子侧链中含苯基 的 $\mathbf{2 a}$ 光照 $100 \mathrm{~min}$ 吸光度下降不到 3\%, 这说明, 引入 苯基有利于增强光化学稳定性. 这也许是为何其他作者 要制备含苯基取代的化合物 1 的原因.

\subsection{2 在 PMMA 中的光化学稳定性}

化合物 2a 2 d 在 PMMA 中(质量分数: $0.03 \mathrm{wt} \%$ ) 的光化学稳定性测试结果见图 8. 相比于在溶液中, 在 同样的光照条件下, 化合物 $\mathbf{2 a} \sim \mathbf{2 d}$ 在 PMMA 中的光化 学稳定性要高于其在甲苯中的光化学稳定性. 光照 100 $\min$, 即使是化合物 $\mathbf{2 b}$ 的吸光度下降也不到 $2 \%$. 这说 明, 化合物 2a $\sim 2 \mathbf{d}$ 掺杂在固体的 PMMA 中光化学稳定

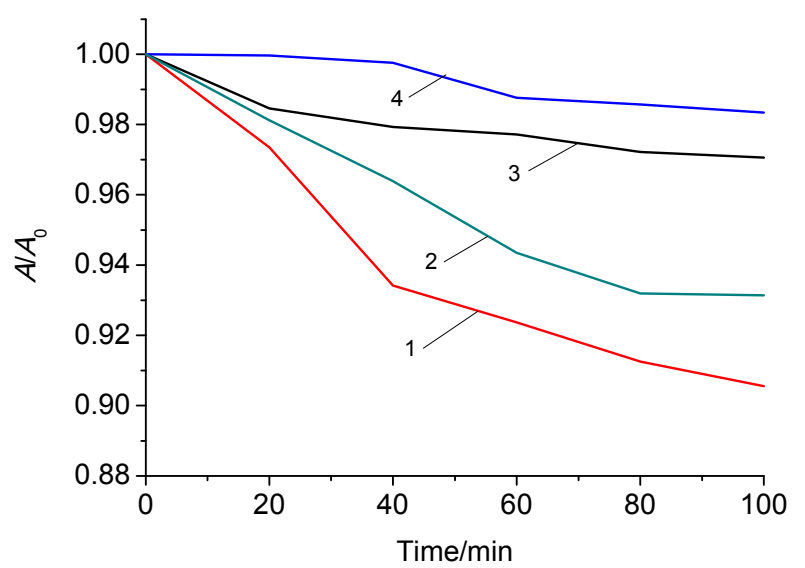

图 7 化合物 $\mathbf{2 a} \sim 2 \mathrm{~d}$ 在甲苯中的光稳定性

Figure 7 Photo-stability of compound $\mathbf{2 a} \sim \mathbf{2 d}$ in toluene (1) 2b, (2) 2d, (3) 2a, (4) 2c

性会提高，这也许是因为当染料处于固态介质中时，它 的活动范围在受到固态聚合物束缚的同时也受到聚合 物的保护，降低了受单线态氧的攻击程度.

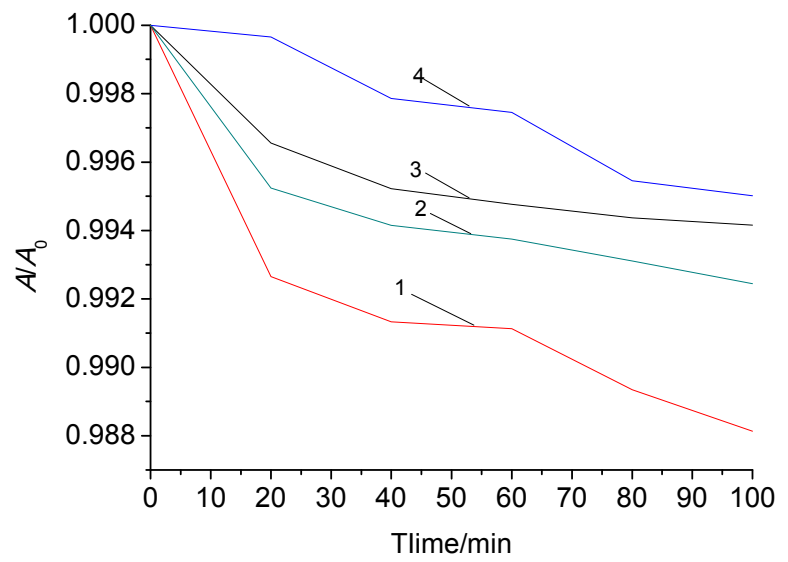

图 8 化合物 $\mathbf{2 a} \sim 2 \mathrm{~d}$ 在 PMMA 中的光稳定性能

Figure 8 Photo-stability of compounds $\mathbf{2 a} \sim \mathbf{2 d}$ in PMMA (1) 2b, (2) 2d, (3) 2a, (4) 2c

\section{2 结论}

合成了一类侧链引入不饱和基团的硫代双烯型镍 络合物, 它们在非极性溶剂(甲苯)中的最大吸收波长在 $1000 \sim 1010 \mathrm{~nm}$ 之间，在固态 PMMA 中的吸收波长也在 $1000 \mathrm{~nm}$ 左右. 颇具意外的是, 取代基的不同对它们最 大吸收波长位移的影响很小，同时它们在丙酮中的吸光 度要明显低于它们在甲苯中的吸光度. 在相同的碳链中 引入苯基有助于提高这类化合物的热稳定性、光化学稳 定性和电化学稳定性. 将它们掺入到 PMMA 中制得的 近红外滤光片, 在阻隔近红外光的同时, 在可见光区域 的吸收很小, 同时其光化学稳定性也高于它们在溶液中 的光化学稳定性，适用于制作护目镜之类的防护红外激 
光的器械.

\section{3 实验部分}

\section{1 仪器与试剂}

${ }^{1} \mathrm{H}$ NMR 核磁共振由 Bruker AVANCE400 核磁共振 仪测定, TMS 为内标; 质谱由 LC/TOF 和 MALPI TOF 质谱仪测定; 循环伏安曲线 $(\mathrm{CV})$ 由 Zahner 电化学工作 站测定, 铂电极作为对电极, 饱和甘录作为参比电极, 硫酸钠 $(0.5 \mathrm{~mol} / \mathrm{L})$ 为电解液, 扫描速度为 $100 \mathrm{mV} / \mathrm{s}$; 热 重分析采用 Netzsch STA 409 PC/PG 热重分析仪, 在氮 气氛围中, 以 $10{ }^{\circ} \mathrm{C} / \mathrm{min}$ 的升温速率测定, 测试温度范 围为 $200 \sim 600{ }^{\circ} \mathrm{C}$; 吸收光谱由 SHMADZU UV-2450 紫 外-可见-近红外光谱仪测定; 溶液的光化学稳定性在光 化学反应器(Beijing Trustech PLS-SXE300c)中进行, 300 瓦氙灯为光源, 测试时, 将化合物 $\mathbf{2 a} \sim \mathbf{2 d}$ 溶解在甲苯 中, 浓度 $1.00 \times 10^{-4} \mathrm{~mol} / \mathrm{L}$, 固体滤色片的光化学稳定 性以 300 瓦氙灯为光源, 测试时将样品(含 $0.03 \mathrm{wt} \%$ 的 PMMA 滤色片)放置在距离氙灯 $10 \mathrm{~cm}$ 处.

锌酸盐按照文献[18]的方法合成, 其他化学品均为 市售分析纯或化学纯试剂, 未经精制直接使用. 光谱测 试用的溶剂经过减压蒸馏处理. 制作滤色片用的聚甲基 丙烯酸甲酯(PMMA)购自阿拉丁试剂公司.

\section{2 合成}

3.2.14,5-二(4'-叔丁基苄基)硫-1,3-二硫杂环戊烯-2硫酮 $(4 a)$ 的合成

将锌酸盐 $(8.60 \mathrm{~g}, 12 \mathrm{mmol})$ 和乙腈 $(100 \mathrm{~mL})$ 加到三 口烧瓶中, 加热到回流, 滴加 4-叔丁基茮溴(13.62 g, 60 $\mathrm{mmol}$ )的乙腈溶液, TLC 跟踪反应进程. 反应结束后, 冷 却, 过滤, 滤饼用乙腈洗涤. 合并滤液和洗涤液, 浓缩 后硅胶柱层析分离提纯[洗脱剂: $V$ (石油醚)： $V$ (二氯甲 烷 $)=6: 1$ ], 得黄色油状物 $4 a$. 产率 $84.1 \%$. ${ }^{1} \mathrm{H} \mathrm{NMR}$ $\left(400 \mathrm{MHz}, \mathrm{CDCl}_{3}\right) \delta: 7.39 \sim 7.32(\mathrm{~m}, 8 \mathrm{H}), 7.18$ (d, $J=8.3$ $\mathrm{Hz}, 8 \mathrm{H}), 3.91$ (s, 8H), 1.31 (s, 36H); MS m/z: $490\left(\mathrm{M}^{+}\right)$.

$4 \mathrm{~b}$ 和 $4 \mathrm{c}$ 的反应类似 $4 \mathrm{a}$.

4,5-二(烯丙基)硫-1,3-二硫杂环戊烯-2-硫酮(4b): 产率 84.6\%, ${ }^{1} \mathrm{H}$ NMR $\left(400 \mathrm{MHz}, \mathrm{CDCl}_{3}\right) \delta: 5.97 \sim 5.67$ $(\mathrm{m}, 4 \mathrm{H}), 5.37 \sim 5.00(\mathrm{~m}, 8 \mathrm{H}), 3.64 \sim 3.26(\mathrm{~m}, 8 \mathrm{H})$; MS $m / z: 278\left(\mathrm{M}^{+}\right)$.

4,5-二(3'-苯基-烯丙基)硫-1,3-二硫杂环戊烯-2-硫酤 (4c): 产率 $85.8 \%,{ }^{1} \mathrm{H} \mathrm{NMR}\left(400 \mathrm{MHz}, \mathrm{CDCl}_{3}\right) \delta: 7.33$ (dd, $J=19.2,9.5 \mathrm{~Hz}, 8 \mathrm{H}), 7.14(\mathrm{dd}, J=29.9,7.7 \mathrm{~Hz}, 8 \mathrm{H})$, $6.69(\mathrm{dd}, J=17.5,10.9 \mathrm{~Hz}, 4 \mathrm{H}), 5.75$ (d, $J=17.6 \mathrm{~Hz}, 4 \mathrm{H})$, 5.27 (dd, $J=10.8,5.9 \mathrm{~Hz}, 4 \mathrm{H}), 3.94 \sim 3.80$ (m, 8H); MS $m / z: 430\left(\mathrm{M}^{+}\right)$.
3.2.2 4,5-二(癸基)硫-1,3-二硫杂环戊烯-2-硫酮 (4d) 的合成

将锌酸盐(8.60 g, $12 \mathrm{mmol})$ 和乙腈 $(100 \mathrm{ml})$ 加到三口 烧瓶中, 加热到回流, 滴加溴癸烷(13.26 g, 60 mmol)的 乙腈溶液, TLC 跟踪反应进程. 反应结束后, 冷却, 过 滤，滤饼用乙腈洗涤. 合并滤液和洗涤液，浓缩后硅胶 柱层析分离提纯, (石油醚作为洗脱剂), 得黄色油状物, 产率 87.2\%. ${ }^{1} \mathrm{H}$ NMR $\left(400 \mathrm{MHz}, \mathrm{CDCl}_{3}\right) \delta: 2.89$ (t, $J=7.3$ $\mathrm{Hz}, 8 \mathrm{H}), 1.78 \sim 1.61(\mathrm{~m}, 8 \mathrm{H}), 1.40(\mathrm{~d}, J=23.2 \mathrm{~Hz}, 8 \mathrm{H})$, $1.29(\mathrm{~s}, 48 \mathrm{H}), 0.91(\mathrm{t}, J=6.5 \mathrm{~Hz}, 12 \mathrm{H}) ; \mathrm{MS} \mathrm{m} / z: 478\left(\mathrm{M}^{+}\right)$. 3.2.3 侧链含不饱和基团取代的硫代双烯型金属镍 络合物 $2 \mathrm{a} \sim \mathbf{2 c}$ 的合成

4,5-二硫代-4-叔丁基苠基-1,3-二硫杂环戊烯-2-硫 酮(4a) $(10 \mathrm{mmol})$ 加入到新鲜配制的甲醇钠 $(25 \mathrm{mmol}) /$ 甲 醇中. 在 $65{ }^{\circ} \mathrm{C}$ 反应, 当反应液的颜色呈棕黄色时停止 反应(约 $2.5 \mathrm{~h}$ ). 冷却到室温, 滴加 $n-\mathrm{Bu}_{4} \mathrm{NBr}(3.22 \mathrm{~g}, 10$ $\mathrm{mmol})$ 和 $\mathrm{NiCl}_{2} \bullet 6 \mathrm{H}_{2} \mathrm{O}(1.19 \mathrm{~g}, 5 \mathrm{mmol})$ 的甲醇溶液, 室温 反应 $5 \mathrm{~h}$, 减压蒸出甲醇, 残余物用乙腈 $(100 \mathrm{~mL})$ 溶解 后, 加入 $\mathrm{I}_{2}(3.81 \mathrm{~g}, 15 \mathrm{mmol})$, 室温下反应过夜. 过滤, 分别用甲醇和二氯甲烷洗涤，合并用二氯甲烷洗涤的洗 涤液, 旋蒸出溶剂, 残余物用硅胶柱层析分离洗脱剂: $V$ (石油醚) $: V$ (二氯甲烷 $)=6 ： 1]$ 得目标化合物 $\mathbf{2 a}$, 产率 $34.2 \%$. ${ }^{1} \mathrm{H}$ NMR (400 MHz, $\mathrm{CDCl}_{3}$ ) $\delta: 7.35$ (s, 16H), 4.53 $(\mathrm{s}, 8 \mathrm{H}), 1.31(\mathrm{~s}, 36 \mathrm{H})$. HRMS calcd for $\mathrm{C}_{48} \mathrm{H}_{60} \mathrm{NiS}_{8}$ 950.1814, found 950.1962 .

$2 b$ 和 $2 \mathrm{c}$ 的反应类似 $2 \mathrm{a}$.

2b: 产率 $32.7 \%$. ${ }^{1} \mathrm{H}$ NMR $\left(400 \mathrm{MHz}, \mathrm{CDCl}_{3}\right) \delta$ : $6.07 \sim 5.97(\mathrm{~m}, 4 \mathrm{H}), 5.40(\mathrm{~d}, J=16.0,4 \mathrm{H}), 5.24(\mathrm{~d}, J=$ $16.0,4 \mathrm{H}), 4.03(\mathrm{~d}, J=7.2 \mathrm{~Hz}, 8 \mathrm{H})$. HRMS calcd for $\mathrm{C}_{16} \mathrm{H}_{20} \mathrm{NiS}_{8}$ 525.8684, found 525.8748.

2c: 产率 $31.6 \%$. ${ }^{1} \mathrm{H}$ NMR $\left(400 \mathrm{MHz}, \mathrm{CDCl}_{3}\right) \delta$ : $7.44 \sim 7.26(\mathrm{~m}, 20 \mathrm{H}), 6.70 \sim 6.74(\mathrm{~m}, 4 \mathrm{H}), 5.75(\mathrm{~d}, J=$ $20.0 \mathrm{~Hz}, 4 \mathrm{H}), 5.26$ (d, $J=12.0 \mathrm{~Hz}, 4 \mathrm{H}), 4.53$ (s, 4H). HRMS calcd for $\mathrm{C}_{400} \mathrm{H}_{36} \mathrm{NiS}_{8} 829.9936$, found 830.0045.

3.2.4 侧链含饱和基团取代的硫代双烯型金属镍络 合物 $2 \mathbf{d}$ 的合成

将 4,5-二硫代癸基-1,3-二硫杂环戊烯-2-硫酮(4d) (10 mmol)加入到新鲜配制的甲醇钠 $(25 \mathrm{mmol}) /$ 甲醇中. 在 $75{ }^{\circ} \mathrm{C}$ 反应，当反应液的颜色呈棕黄色时停止反应(约 $3 \mathrm{~h})$. 冷却到室温, 滴加 $n-\mathrm{Bu}_{4} \mathrm{NBr}(3.22 \mathrm{~g}, 10 \mathrm{mmol})$ 和 $\mathrm{NiCl}_{2} \cdot 6 \mathrm{H}_{2} \mathrm{O}(1.19 \mathrm{~g}, 5 \mathrm{mmol})$ 的甲醇溶液, 室温反应 $5 \mathrm{~h}$, 减压蒸出甲醇, 残余物用乙腈 $(100 \mathrm{~mL})$ 溶解后, 加入 $\mathrm{I}_{2}$ (3.81 g, $15 \mathrm{mmol})$, 室温下反应过夜. 过滤, 分别用甲醇 和二氯甲烷洗涤，合并用二氯甲烷洗涤的洗涤液，旋蒸 出溶剂, 残余物用硅胶柱层析分离 [洗脱剂: $V($ 石油 
醚)： $V($ 二氯甲烷 $)=6 ： 1$ 得目标化合物 $2 \mathbf{d}^{[2]}$, 产率 35.8\%. ${ }^{1} \mathrm{H}$ NMR (400 MHz, $\left.\mathrm{CDCl}_{3}\right) \delta: 3.40 \sim 3.36(\mathrm{~m}$, $5 \mathrm{H}), 1.87 \sim 1.84(\mathrm{~m}, 5 \mathrm{H}), 1.50(\mathrm{~s}, 62 \mathrm{H}), 1.27(\mathrm{~s}, 62 \mathrm{H})$, $0.89 \sim 0.86(\mathrm{~m}, 12 \mathrm{H})$; MS $m / z: 926.3\left(\mathrm{M}^{+}\right)$.

\section{3 近红外滤光片的制作}

将 $5.0 \mathrm{mg}$ 化合物 $\mathbf{2 a} \sim 2 \mathbf{d}$ 分别溶于 $\mathrm{CHCl}_{3}$, 在 $50 \mathrm{~mL}$ 容量瓶中定容. 用移液管分别移取 4、8、12 和 $16 \mathrm{~mL}$ 溶液于小烧瓶中, 并向每份溶液中加入 $4 \mathrm{~g}$ 聚甲基丙烯 酸甲酯(PMMA)和 1 滴塑化剂(邻苯二甲酸二丁酯), 室 温下搅拌至 PMMA 完全溶解. $4 \mathrm{~h}$ 后, 将溶液倒入同一 规格的培养血内, 待溶剂挥发后即得质量分数分别为 $0.01 \sim 0.04 \mathrm{wt} \%$ 的近红外吸收滤光片.

辅助材料(Supporting Information) 化合物 $\mathbf{2 a} \sim 2 \mathrm{~d}$ 的 核磁共振氢谱和质谱谱图. 这些材料可以免费从本刊网 站(http://sioc-journal.cn/)上下载.

\section{References}

[1] Men, J. F.; Cheng, H. F.; Chen, Z. H.; Chu, Z. Y. Mater. Rev. 2008, 22, 3 (in Chinese). (门金风, 程海峰，陈朝辉，楚增勇，材料导报， 2008，22, 3.)

[2] Marshall, K. L.; Painte, G.; Lotito, K. A. Mol. Cryst. Liquid Cryst. 2006, 47,449 .

[3] Aragoni, C.; Arca, M.; Demartin, F.; Devillanova, F. A.; Garau, A.; Isaia, F.; Lelj, F.; Lippolis, V.; Verani, G. J. Am. Chem. Soc. 1991, $121,7098$.

[4] Mueller-Westerhoff, U. T.; Vance, B.; Yoon, D. I. Tetrahedron 1991, 47, 909.

[5] Espine, P. Coord. Chem. Rev. 1992, 117, 215.
[6] Fabian, J.; Nakazumi, H.; Matsuoka, M. Chem. Rev. 1992, 92(6), 1200.

[7] Coe, B. J. Compr. Coord. Chem. 2004, 9, 621.

[8] Cho, J. Y.; Domercq, B.; Jones, S. C.; Yu, J.; Zhang, X.; An, Z.; Bishop, M.; Barlow, S.; Marder, S. R.; Kippelen, B. J. Mater. Chem. 2007, 17, 2642.

[9] Stiefel, E. I. Karlin, K. D. Dithiolene Chemistry, Progress in Inorganic Chemistry, John Wiley Sons, 2004, 52, 399.

[10] Deplano, P.; Pilia, L.; Espa, D.; Mercuri, M. L.; Serpe, A. Coord. Chem. Rev. 2010, 254, 1434.

[11] Robertson, N.; Cronin, L. Coord. Chem. Rev. 2002, 227, 93.

[12] Chen, H.; Cheng, Z. S. J. East China Institute Chem. Technol. 1992, 18(5), 628 (in Chinese). (陈红, 程铸生, 华东化工学院学报, 1992, 18(5), 628.)

[13] Men, J. F.; Cheng, H. F.; Chen, Z. H.; Chu, Z. Y.; Zheng, W. W.; Wang, Q. Infrared Technol. 2008, 30, 150 (in Chinese). (门金风, 程海峰, 陈朝辉, 楚增勇, 郑文伟, 王茜, 红外技术, 2008, 30, 150.)

[14] Dai, F.; Fan, W. H.; Wu, J. H.; Wang, L. X.; Zhang, Q. T. Laser Infrared 2011, 41, 1131 (in Chinese).

(戴峰，樊卫华，吴金华，王丽熙，张其土，激光与红外，2011， 41(10), 1131.)

[15] Gao, J. X.; Miao, Q. Q.; Wang, Z. Q.; Gao, Y. R.; Ma, Y. L. Fine Chem. 2011, 28, 909 (in Chinese).

(高俊雄, 苗青青, 王泽清, 高玉荣, 马廷丽, 精细化工, 2011, 28, 909.)

[16] Charlton, A.; Hill, C. A. S.; Underhill, A. E.; Malik, K. M. A.; Hursthouse, M. B.; Karaulovb, A. I.; Meller, J. J. Mater. Chem. 1994, 4(12), 1861.

[17] Robertson, N.; Cronin, L. Coord. Chem. Rev. 2002, 227, 93.

[18] Svenstrup, N.; Becher, J. Synthesis 1995, 215.

[19] Bui, T. T.; Vuong, M. H. New J. Chem. 2012, 36, 2033.

[20] Huang, Q.; Wang, L. X.; Zhang, Q. S. Laser Infrared 2008, 38(5), 421 (in Chinese).

(黄强, 王丽熙, 张其土, 激光与红外, 2008, 38(5), 421.) 